\title{
BDNF Val66Met polymorphism in patterns of neural activation in individuals with MDD and healthy controls
}

Danuta M. Lisiecka ${ }^{1,2^{*}}$, Erik O’Hanlon ${ }^{3}$, Andrew J. Fagan ${ }^{4,5}$, Angela Carballedo ${ }^{6}$, Derek

Morris $^{7}$, John Suckling ${ }^{1,2,8}$, Thomas Frodl ${ }^{6,9}$

${ }^{1}$ Brain Mapping Unit, Department of Psychiatry, University of Cambridge, UK

${ }^{2}$ Behavioural and Clinical Neuroscience Institute, Department of Psychiatry, University of

Cambridge, UK

${ }^{3}$ Department of Psychiatry, Faculty of Medicine and Health Sciences, Royal College of

Surgeons, Dublin, Ireland

${ }^{4}$ Centre for Advanced Medical Imaging (CAMI), St James's Hospital, Dublin, Ireland

${ }^{5}$ Department of Clinical Medicine, Trinity College Dublin, Ireland

${ }^{6}$ Department of Psychiatry, Trinity College Dublin, Ireland

${ }^{7}$ Cognitive Genetics and Therapy Group, Discipline of Biochemistry and School of

Psychology, National University of Ireland, Galway, Ireland

${ }^{8}$ Cambridge and Peterborough NHS Foundation Trust, Cambridge, UK

${ }^{9}$ Department of Psychiatry, University of Regensburg, Germany

\footnotetext{
* Correspondence address:

Dr Danuta M. Lisiecka

Brain Mapping Unit

Department of Psychiatry

University of Cambridge

Herchel Smith Building for Brain and Mind Sciences

Robinson Way

Cambridge

CB2 0SZ

UK
}

Email address: $\underline{\text { dml45@cam.ac.uk }}$

Telephone: +44 1223336966

Fax: +44 1223336581* 
Keywords: major depressive disorder, brain-derived neurotrophic factor, functional magnetic resonance imaging, emotional response, striatum, prefrontal cortex 


\section{Abstract}

\section{Background}

Rs6265 single nucleotide polymorphism, which influences brain-derived neurotrophic factor (BDNF) levels in the cortical and subcortical brain structures, may result in distinguished patterns of neural activation during a major depressive disorder (MDD) episode. Valine homozygotes with high levels of BDNF and methionine carriers with lower levels of BDNF may present specific neural correlates of MDD. In our study we have tested differences in blood oxygen level dependent (BOLD) signal between individuals with MDD and healthy controls for both allelic variants.

\section{Methods}

Individuals with MDD (N=37) and healthy controls ( $=39)$ were genotyped for rs6265 and compared separately in each allelic variant for BOLD response in a functional magnetic resonance imaging experiment examining appraisal of emotional scenes. The two allelic variants were also compared separately for both individuals with MDD and healthy controls.

\section{Results}

In the homozygous valine group MDD was associated with decreased neural activation in areas responsible for cognitive appraisal of emotional scenes. In the methionine group MDD was related to increased activation in subcortical regions responsible for visceral reaction to emotional stimuli. During an MDD episode methionine carriers showed more activation in areas associated with cognitive appraisal of emotional information in comparison to valine homozygotes.

\section{Limitations}

Small sample size of healthy controls carrying methionine $(\mathrm{N}=8)$. 


\section{Conclusion}

Our results suggest that allelic variations in the rs6265 gene lead to specific neural correlates of MDD which may be associated with different mechanisms of MDD in the two allelic groups. This may have potential importance for screening and treatment of patients. 


\section{Introduction}

According to the neuroplasticity theory, brain-derived neurotrophic factor (BDNF) ${ }^{*}$, a peptide responsible for neuronal growth, plays a significant role in the pathogenesis of major depressive disorder (MDD) (Brunoni et al., 2008; Castren et al., 2007; Groves, 2007). BDNF is associated with plasticity and long-term synaptic connectivity in neural networks including the hippocampus, frontal lobes and striatum (Fossati et al., 2004). Altered secretion of BDNF observed in MDD can lead to long-term changes in these regions and consequently to cognitive symptoms characteristic of MDD (Fossati et al., 2004). An increase of BDNF level in specific neural areas can either promote or inhibit an antidepressant-like reaction (Castren et al., 2007; Groves, 2007).

In rodents a direct injection of BDNF into the hippocampus diminishes depressive symptoms (Shirayama et al., 2002). Patients with MDD have reduced volumes of the hippocampus and frontal lobes (Dwivedi et al., 2003; Knable et al., 2004; Stockmeier et al., 2004), a sign of neuronal atrophy associated with lower levels of BDNF (Martinowich et al., 2007). One mechanism of action of therapies for MDD involves an increase of BDNF concentration in the hippocampus and frontal lobes (Brunoni et al., 2008). Conversely, a high concentration of BDNF in the midbrain is depressogenic and in rodents its reduction eliminates a depressivelike behaviour (Berton et al., 2006; Eisch et al., 2003).

Substituting valine by methionine in the Val66Met BDNF gene (rs6265) reduces activitydependent secretion of BDNF (Chen et al., 2004). This single-nucleotide polymorphism has

\footnotetext{
*Abbreviations:

BDNF - brain-derived neurotrophic factor

MDD - major depressive disorder

HDRS - Hamilton Depression Rating Scale

MADRS - Montgomery Asberg Depression Rating Scale

BDI-II - Beck Depression Inventory II

SNP - single nucleotide polymorphism

$f \mathrm{MRI}$ - functional magnetic resonance imaging

IAPS - International Affective Picture System

$\mathrm{RT}$ - reaction time

DLPFC - dorsolateral prefrontal cortex
} 
been previously associated with MDD (Binder and Scharfman, 2004). Carriers of at least one methionine show increased depressive behaviours (Schumacher et al., 2005), while displaying reduced volumes of frontal lobes (Nemoto et al., 2006; Pezawas et al., 2004) and hippocampus (Bueller et al., 2006; Pezawas et al., 2004), similar to patients with MDD (Koolschijn et al., 2009). Conversely, homozygous valine individuals score higher on the neuroticism scale, which is symptomatic of liability to MDD (Hunnerkopf et al., 2007). This suggests that a different neuronal pathway may lead to developing MDD in the two genotypes.

Our study compares healthy controls and patients with MDD separately for both genotypes in neural correlates of emotional appraisal. Emotional appraisal is a part of the emotional regulation system monitoring approach-withdrawal behaviour, and in patients with MDD it could be altered in two ways. Firstly, according to emotional potentiation theory, patients with MDD would show a stronger visceral reaction during emotional appraisal (Bylsma et al., 2008). Alternatively, according to emotion context insensitivity theory, patients with MDD would be less-reactive to emotional stimuli (Rottenberg et al., 2005). Both of these theories are associated with emotional flexibility connected with the role of BDNF in the brain.

\section{Methods}

\section{Subjects}

Thirty-seven patients with MDD and thirty-nine healthy controls - matched for age, gender and handedness (all right-handed) - participated in the study. Written informed consent was obtained from all study participants. The study protocol was approved by the local ethics committee of Trinity College Dublin, Ireland, and prepared in accordance to the ethical standards of the Declaration of Helsinki. 
Participants with MDD were recruited from the Adelaide and Meath Hospital incorporating the National Children's Hospital, Dublin, and from the St James's Hospital, Dublin. The MDD diagnoses and lack of comorbidities for all the subjects were confirmed by the consensus of two consultant psychiatrists, one examining participants prior to the study and the other performing the structured Clinical Interview for Diagnostic Statistics Manual IV (First and Gibbon, 2004) two to three days prior to the assessment day. The medical exclusion criteria of the study included: previous or present head injury; a current or past psychiatric or neurological disease (apart from MDD in the case of the patient group); current medical disease influencing the nervous system; substance dependency. On the assessment day, participants' health was verified via the Hamilton Depression Rating Scale (HDRS), Montgomery Asberg Depression Rating Scale (MADRS) and Beck Depression Inventory II (BDI-II). Depressed individuals differed significantly from healthy controls in all depression ratings (Lisiecka et al., 2013a). All healthy participants scored within the norm interval in HDRS, MDRS and BDI-II, and all subjects with MDD scored over the threshold characteristic for disease. Among patients, twelve were not medicated, thirteen were being treated with selective serotonin reuptake inhibitors and twelve with dual action substances. Five patients had had one previous episode of MDD, two patients had two previous episodes of MDD, while the remaining group were experiencing a first episode of MDD.

Both patients with MDD and healthy controls were genotyped for the variants of the rs6265 single nucleotide polymorphism (SNP). The Val66Met BDNF SNP (rs6265) was genotyped in the sample using a Taqman ${ }^{\circledR}$ SNP Genotyping Assay on a 7900HT Sequence Detection System (Applied Biosystems). The call rate for the Taqman genotyping was $>95 \%$, and all samples were in Hardy-Weinberg equilibrium ( $>>0.05)$. Along with the test samples, a number of HapMap CEU DNA sample positive controls (www.hapmap.org) and nontemplate negative controls were genotyped for each SNP for quality control purposes. For 
positive controls, all genotypes were found to be concordant with the available online HapMap data. All non-template samples returned a negative result. As a result, participants were divided into three genotype groups: homozygous groups for methionine (bdnfmet66met) and valine alleles (bdnfval66val), and a heterozygous group carrying one methionine and one valine allele (bdnfval66met). Heterozygous individuals and subjects with two methionine alleles were combined into one group of methionine-carriers (bdnfval66metor-bdnfmet66met), as has been done in previous functional magnetic resonance imaging (fMRI) studies investigating Val66Met polymorphism (Lau et al., 2010; Montag et al., 2008) since methionine homozygotes are infrequent (Shimizu et al., 2004), and having at least one methionine allele is associated with susceptibility to MDD (Frodl et al., 2007). After the diagnostic and genotyping procedure, four groups of participants were distinguished: bdnfval66val patients with MDD (N=23; age $=41.3 \pm 12.4$ years; 17 females and 6 males; HDRS=28.8 \pm 6.5 ; MADRS=29.6 \pm 7.4 ; BDI-II=30.7 \pm 12.2 ), bdnfval66met-orbdnfmet66met patients with MDD $(\mathrm{N}=14$; age $=41.9 \pm 8.1$ years; 9 females and 5 males; HDRS=28.3 $\pm 6.9 ;$ MADRS=29.4 $\pm 4.9 ;$ BDI-II=35.7 \pm 10.6 ), bdnfval66val healthy controls $(\mathrm{N}=31 ;$ age $=38.3 \pm 13.3$ years; 19 females and 12 males; $\mathrm{HDRS}=2.6 \pm 2.4 ; \mathrm{MADRS}=1.5 \pm 2.6$; BDI-II=1.9 \pm 2.3$)$, and bdnfval66met-or-bdnfmet66met healthy carriers $(\mathrm{N}=8$; age $=33.5 \pm 14.1$ years; 4 females and 4 males; HDRS $=2.3 \pm 1.7$; MADRS=3.3 \pm 4.8 ; BDI-II $=4.3 \pm 4.9)$. The groups did not differ in age $(\mathrm{p}=0.369)$ or gender $(\mathrm{p}=0.623)$. They differed in all applied ratings of MDD (HDRS $\mathrm{p}<0.001$; MADRS $\mathrm{p}<0.001$; BDI-II $\mathrm{p}<0.001$ ). However, there was no significant difference in the depression ratings between the two groups of healthy controls (HDRS $\mathrm{p}=0.998 ;$ MADRS $\mathrm{p}=0.82 ;$ BDI-II $\mathrm{p}=0.304$ ) and between the two groups of MDD patients (HDRS p=0.988; MADRS $\mathrm{p}=1$; BDI-II $\mathrm{p}=0.898$ ). Furthermore, the two patients groups did not differ in the method of treatment $(\mathrm{p}=0.914)$ and the number of previous episodes of MDD ( $\mathrm{p}=0.397)$. 


\section{Design}

The study utilized a four sample design with bdnfval66val patients with MDD, bdnfval66met-or-bdnfmet66met patients with MDD, bdnfval66val healthy controls, and bdnfval66met-or-bdnfmet66met healthy controls as comparison groups. An event-related fMRI experiment measuring emotional appraisal of scenes with standardized ratings of emotional valence from International Affective Picture System (IAPS) was used during the recording of blood oxygen level dependent signals for each subject (Lang et al., 1999). The pictures selected for the study represented a broad range of emotional valence: from 1 (very negative) to 9 (very positive) on the IAPS valence scale, and were evenly distributed across the scale. To ensure that the chosen pictures had a consistent appraisal in the healthy population, those with minimal standard deviation in emotional valence and the ones judged similarly by men and women were selected for the task. The content of the pictures presented both people and objects. The details of the task are presented in Lisiecka et al. (Lisiecka et al., 2013b).

\section{Analysis}

A 4-group MANOVA was calculated to determine whether the groups differed in respect to reaction times (RTs) and accuracy measures. Subsequently, a post-hoc analysis was performed for participants' RTs and accuracy.

A 2x2 factorial analysis was performed with the diagnosis of MDD as the first and the Val66Met gene allele as the second factor. Participants' age and gender were added as covariates of no interest. Contrasts were calculated to determine the differences between bdnfval66val healthy controls and bdnfval66met-or-bdnfmet66met healthy controls, between bdnfval66val healthy controls and bdnfval66val patients with MDD, between bdnfval66metor-bdnfmet66met healthy controls and bdnfval66met-or-bdnfmet66met patients with MDD, and between bdnfval66val patients with MDD and bdnfval66met-or-bdnfmet66met patients 
with MDD. The regions surviving the whole-brain cluster-level analysis with false discovery rate correction for multiple comparisons at $\mathrm{p}<0.05$ are presented in results section. Automated anatomical labelling was used to localize the significant results in a standard stereotactic space (template - the Montreal Neurological Institute).

\section{Results}

\section{Behavioural results}

Bdnfval66val healthy controls displayed accuracy of $83.7 \pm 8.1 \%$ with RT of $1.33 \pm 0.32$ seconds. For other groups the results were as followed: bdnfval66val patients with MDD accuracy $=74.8 \pm 13 \%, \mathrm{RT}=1.5 \pm 0.32$ seconds; bdnfval66met-or-bdnfmet66met patients with MDD - accuracy $=76.9 \pm 10.5 \%, \mathrm{RT}=1.64 \pm 0.58$ seconds; bdnfval66met-or-bdnfmet66met healthy controls - accuracy $=79.3 \pm 16.2 \%, \mathrm{RT}=1.36 \pm 0.31$ seconds. The only difference discovered by behavioural analysis was the diminished accuracy of bdnfval66val MDD patients in comparison to bdnfval66val healthy controls in emotional appraisal $(\mathrm{p}=0.025)$.

\section{$f$ MRI results}

The bdnfval66val healthy controls, in comparison to the bdnfval66val patients with MDD, displayed more neural activation in the left angular gyrus, in the left middle occipital gyrus, in the right postcentral gyrus, in the right middle cingulate gyrus and in the right supramarginal gyrus (Fig. 1A, Tab. 1). The reversed comparison did not show any significant results. The bdnfval66met-or-bdnfmet66met patients with MDD displayed a heightened activation in contrast to the bdnfval66met-or-bdnfmet66met healthy controls in the left caudate nucleus (Fig. 1B, Tab. 1). The reversed comparison did not show any significant results. The bdnfval66met-or-bdnfmet66met healthy controls and the bdnfval66val healthy controls did not differ in neural correlates of emotional processing. The bdnfval66met-or- 
bdnfmet66met patients with MDD experienced more neural activation than the bdnfval66val patients with MDD did in the right middle frontal gyrus and in the right superior frontal gyrus (Fig. 1C, Tab. 1). The reversed comparison did not show any significant results.

\section{Discussion}

We provide evidence that polymorphism in the Val66Met gene, previously associated with BDNF secretion (Chen et al., 2004) and vulnerability to MDD (Brunoni et al., 2008; Castren et al., 2007; Groves, 2007), modulates a difference between patients with MDD and healthy controls in correlates of emotional appraisal. The differences between patients with MDD and healthy controls follow unique pathways for each genotype.

For the bdnfval66val group the differences between patients with MDD and healthy controls concentrate in the cortical compartment of the central nervous system, where patients with MDD experience reduced activation. It suggests a lowered top-down emotional regulation in the bdnfval66val individuals with MDD (Haldane and Frangou, 2006). Such disturbance typically results in a less efficient classification of emotional experiences, as observed in the behavioural responses of the bdnfval66val MDD patients. The areas where these patients displayed a decreased activation in comparison to the bdnfval66val healthy controls during emotional appraisal are involved in an emotional response to visual stimuli (angular and occipital gyri) (Hendler et al., 2001) and recognition of one's own internal affective states (middle cingulate gyrus) (Kober et al., 2008). This suggests that in the bdnfval66val group major depressive disorder is characterized by disturbances in informative aspect of emotional processing.

Conversely, the difference between patients with MDD and healthy controls in the bdnfval66met-or-bdnfmet66met group is localised in a sub-cortical region of striatum, where the patients show higher activation. Striatum participates in the visceral aspect of emotional 
experience (Jensen et al., 2003; Kober et al., 2008). This suggests that bdnfval66met-orbdnfmet66met patients with MDD experience stronger visceral reaction to emotional stimuli (Longstaff, 2005). The increase of activation in striatum (Banner et al., 2011) has been associated with vulnerability to MDD linked to methionine allele. A changed level of BDNF observed in the region can produce depressive-like symptoms (Berton et al., 2006; Eisch et al., 2003). This suggests that MDD in the bdnfval66met-or-bdnfmet66met group may be associated with an atypical distribution of BDNF in this area and thus prolonged stress reaction associated with the hypothalamic-pituitary-adrenal axis (Dawood et al., 2007; Praag et al., 2004).

Finally, the Val66Met polymorphism distinguishes two groups of patients with MDD. In comparison to the bdnfval66val MDD patients, the bdnfval66met-or-bdnfmet66met MDD patients experienced an increased activation in the right dorsolateral prefrontal cortex (DLPFC). The DLPFC has a strong connection with BDNF but should not be activated during emotional appraisal (Berton et al., 2006; Eisch et al., 2003; Nemoto et al., 2006; Pezawas et al., 2004). However, its increased activation in the bdnfval66met-orbdnfmet66met MDD patients may further indicate their incorrect BDNF distribution or be a symptom of diminished top-down regulation in the bdnfval66val MDD patients. Interestingly, there was no difference observed in amygdalae, areas typically associated with emotional appraisal. However, the nature of our task (event-related) as well as the themes of visual stimuli used (combination of objects and people) could account for this result (Sergerie et al., 2008).

\section{Limitations}

Our analysis was performed as a sub-section of a larger study investigating the influence of the family history of depression on emotional processing in individuals with MDD and healthy controls (Lisiecka et al., 2013a). This is why the numbers of participants in the 
groups in the present analysis may not have been optimal. However, methionine-allele in rs6265 is rare in the human population and studies of the methionine carrying group often show a disproportion between numbers of individuals with methionine and valine alleles.

\section{Conclusion}

The BDNF gene has been shown to differentiate effectively between different sub-types of patients with MDD. This study reports neural differences in emotional appraisal between bdnfval66val patients with MDD and bdnfval66met-or-bdnfmet66met patients. The patients with two different genetic backgrounds differed in neither clinical features nor behavioural performance. However, the differences in emotional processing between the sub-types of patients with MDD suggest that the two groups follow unique trajectories towards MDD. This indicates the potential role genetic screening may play in choosing the appropriate treatment for different types of MDD, thus offering more effective treatment outcomes. 


\section{Conflict of interest}

All authors declare no conflict of interest.

\section{Acknowledgements}

Funding for this study was provided by the Science Foundation Ireland (Grant Number: SFI/07SK/B1214C). Health Research Board (HRB) Ireland provided funding for magnetic resonance imaging infrastructure at the Centre of Advanced Medical Imaging (CAMI) at St. James's Hospital in Dublin. The authors would like to thank Doctor Izu Ugwu for his help in recruiting the patients and Doctor Matthew Ford for proof-reading the manuscript. The authors would also like to extend their gratitude to Professor Michael Gill for his help in genotyping the cohort.

\section{Contributors}

D.M. Lisiecka designed the experiment, collected the data, analysed the data and wrote the article. E. O'Hanlon reviewed the analysis and the article. A.J. Fagan designed the functional magnetic resonance imaging protocol and reviewed the article. A. Carballedo recruited the participants and collected the data. D. Morris analysed the genetic data. J. Suckling reviewed the article. T. Frodl designed the study, trained the research team, supervised the study and reviewed the article. 


\section{References}

Banner, H., Bhat, V., Etchamendy, N., Joober, R., Bohbot, V.D., 2011. The brain-derived neurotrophic factor Val66Met polymorphism is associated with reduced functional magnetic resonance imaging activity in the hippocampus and increased use of caudate nucleusdependent strategies in a human virtual navigation task. European Journal of Neuroscience 33, 968-977.

Berton, O., McClung, C.A., Dileone, R.J., Krishnan, V., Renthal, W., Russo, S.J., Graham, D., Tsankova, N.M., Bolanos, C.A., Rios, M., Monteggia, L.M., Self, D.W., Nestler, E.J., 2006. Essential role of BDNF in the mesolimbic dopamine pathway in social defeat stress. Science 311, 864-868.

Binder, D.K., Scharfman, H.E., 2004. Brain-derived neurotrophic factor. Growth Factors 22, 123-131.

Brunoni, A.R., Lopes, M., Fregni, F., 2008. A systematic review and meta-analysis of clinical studies on major depression and BDNF levels: implications for the role of neuroplasticity in depression. Int J Neuropsychopharmacol 11, 1169-1180.

Bueller, J.A., Aftab, M., Sen, S., Gomez-Hassan, D., Burmeister, M., Zubieta, J.K., 2006. BDNF Val66Met allele is associated with reduced hippocampal volume in healthy subjects. Biological psychiatry 59, 812-815.

Bylsma, L.M., Morris, B.H., Rottenberg, J., 2008. A meta-analysis of emotional reactivity in major depressive disorder. Clinical psychology review 28, 676-691.

Castren, E., Voikar, V., Rantamaki, T., 2007. Role of neurotrophic factors in depression. Curr Opin Pharmacol 7, 18-21.

Chen, Z.Y., Patel, P.D., Sant, G., Meng, C.X., Teng, K.K., Hempstead, B.L., Lee, F.S., 2004. Variant brain-derived neurotrophic factor (BDNF) (Met66) alters the intracellular trafficking and activity-dependent secretion of wild-type BDNF in neurosecretory cells and cortical neurons. Journal of Neuroscience 24, 4401-4411.

Dawood, T., Anderson, J., Barton, D., Lambert, E., Esler, M., Hotchkin, E., Haikerwal, D., Kaye, D., Lambert, G., 2007. Reduced overflow of BDNF from the brain is linked with suicide risk in depressive illness. Molecular psychiatry 12, 981-983.

Dwivedi, Y., Rizavi, H.S., Conley, R.R., Roberts, R.C., Tamminga, C.A., Pandey, G.N., 2003. Altered gene expression of brain-derived neurotrophic factor and receptor tyrosine kinase B in postmortem brain of suicide subjects. Archives of general psychiatry 60, 804-815. Eisch, A.J., Bolanos, C.A., de Wit, J., Simonak, R.D., Pudiak, C.M., Barrot, M., Verhaagen, J., Nestler, E.J., 2003. Brain-derived neurotrophic factor in the ventral midbrain-nucleus accumbens pathway: a role in depression. Biological psychiatry 54, 994-1005.

First, M.B., Gibbon, M., 2004. The Structured Clinical Interview for DSM-IV Axis I Disorders (SCID-I) and the Structured Clinical Interview for DSM-IV Axis II Disorders (SCID-II). John Wiley \& Sons Inc.

Fossati, P., Radtchenko, A., Boyer, P., 2004. Neuroplasticity: from MRI to depressive symptoms. European Neuropsychopharmacology 14, S503-S510.

Frodl, T., Schule, C., Schmitt, G., Born, C., Baghai, T., Zill, P., Bottlender, R., Rupprecht, R., Bondy, B., Reiser, M., Moller, H.J., Meisenzahl, E.M., 2007. Association of the brainderived neurotrophic factor Val66Met polymorphism with reduced hippocampal volumes in major depression. Archives of general psychiatry 64, 410-416.

Groves, J.O., 2007. Is it time to reassess the BDNF hypothesis of depression? Molecular psychiatry 12, 1079-1088.

Haldane, M., Frangou, S., 2006. Functional neuroimaging studies in mood disorders. Acta Neuropsychiatrica 18, 88-99. 
Hendler, T., Rotshtein, P., Hadar, U., 2001. Emotion-Perception Interplay in the Visual Cortex:"The Eyes Follow the Heart". Cellular and Molecular Neurobiology 21, 733-752. Hunnerkopf, R., Strobel, A., Gutknecht, L., Brocke, B., Lesch, K.P., 2007. Interaction between BDNF Val66Met and dopamine transporter gene variation influences anxiety-related traits. Neuropsychopharmacology : official publication of the American College of Neuropsychopharmacology 32, 2552-2560.

Jensen, J., McIntosh, A.R., Crawley, A.P., Mikulis, D.J., Remington, G., Kapur, S., 2003. Direct activation of the ventral striatum in anticipation of aversive stimuli. Neuron 40, 12511257.

Knable, M.B., Barci, B.M., Webster, M.J., Meador-Woodruff, J., Torrey, E.F., 2004. Molecular abnormalities of the hippocampus in severe psychiatric illness: postmortem findings from the Stanley Neuropathology Consortium. Molecular psychiatry 9, 609-620, 544.

Kober, H., Barrett, L.F., Joseph, J., Bliss-Moreau, E., Lindquist, K., Wager, T.D., 2008. Functional grouping and cortical-subcortical interactions in emotion: a meta-analysis of neuroimaging studies. Neuroimage 42, 998-1031.

Koolschijn, P.C., van Haren, N.E., Lensvelt-Mulders, G.J., Hulshoff Pol, H.E., Kahn, R.S., 2009. Brain volume abnormalities in major depressive disorder: a meta-analysis of magnetic resonance imaging studies. Human Brain Mapping 30, 3719-3735.

Lang, P.J., Bradley, M.M., Cuthbert, B.N., 1999. International affective picture system (IAPS): Technical manual and affective ratings. Gainesville, FL: The Center for Research in Psychophysiology, University of Florida.

Lau, J.Y., Goldman, D., Buzas, B., Hodgkinson, C., Leibenluft, E., Nelson, E., Sankin, L., Pine, D.S., Ernst, M., 2010. BDNF gene polymorphism (Val66Met) predicts amygdala and anterior hippocampus responses to emotional faces in anxious and depressed adolescents. Neuroimage 53, 952-961.

Lisiecka, D.M., Carballedo, A., Fagan, A.J., Ferguson, Y., Meaney, J., Frodl, T., 2013a. Recruitment of the left hemispheric emotional attention neural network in risk for and protection from depression. Journal of psychiatry \& neuroscience : JPN 38, 117-128. Lisiecka, D.M., Carballedo, A., Fagan, A.J., Ferguson, Y., Meaney, J., Frodl, T., 2013b. Recruitment of the left hemispheric emotional attention neural network in risk for and protection from depression. J Psychiatry Neurosci 38, 117-128.

Longstaff, A., 2005. Neuroscience, 2nd ed. Taylor \& Francis Group, New York. Martinowich, K., Manji, H., Lu, B., 2007. New insights into BDNF function in depression and anxiety. Nature neuroscience 10, 1089-1093.

Montag, C., Reuter, M., Newport, B., Elger, C., Weber, B., 2008. The BDNF Val66Met polymorphism affects amygdala activity in response to emotional stimuli: evidence from a genetic imaging study. Neuroimage 42, 1554-1559.

Nemoto, K., Ohnishi, T., Mori, T., Moriguchi, Y., Hashimoto, R., Asada, T., Kunugi, H., 2006. The Val66Met polymorphism of the brain-derived neurotrophic factor gene affects agerelated brain morphology. Neuroscience letters 397, 25-29.

Pezawas, L., Verchinski, B.A., Mattay, V.S., Callicott, J.H., Kolachana, B.S., Straub, R.E., Egan, M.F., Meyer-Lindenberg, A., Weinberger, D.R., 2004. The brain-derived neurotrophic factor val66met polymorphism and variation in human cortical morphology. Journal of Neuroscience 24, 10099-10102.

Praag, H.M.v., Kloet, E.R.d., Os, J.v., 2004. Stress, the brain and depression. Cambridge University Press, Cambridge ; New York.

Rottenberg, J., Gross, J.J., Gotlib, I.H., 2005. Emotion context insensitivity in major depressive disorder. Journal of abnormal psychology 114, 627. 
Schumacher, J., Jamra, R.A., Becker, T., Ohlraun, S., Klopp, N., Binder, E.B., Schulze, T.G., Deschner, M., Schmal, C., Hofels, S., Zobel, A., Illig, T., Propping, P., Holsboer, F., Rietschel, M., Nothen, M.M., Cichon, S., 2005. Evidence for a relationship between genetic variants at the brain-derived neurotrophic factor (BDNF) locus and major depression. Biological psychiatry 58, 307-314.

Sergerie, K., Chochol, C., Armony, J.L., 2008. The role of the amygdala in emotional processing: a quantitative meta-analysis of functional neuroimaging studies. Neuroscience \& Biobehavioral Reviews 32, 811-830.

Shimizu, E., Hashimoto, K., Iyo, M., 2004. Ethnic difference of the BDNF 196G/A (val66met) polymorphism frequencies: the possibility to explain ethnic mental traits. American Journal of Medical Genetics Part B: Neuropsychiatric Genetics 126, 122-123.

Shirayama, Y., Chen, A.C., Nakagawa, S., Russell, D.S., Duman, R.S., 2002. Brain-derived neurotrophic factor produces antidepressant effects in behavioral models of depression. Journal of Neuroscience 22, 3251-3261.

Stockmeier, C.A., Mahajan, G.J., Konick, L.C., Overholser, J.C., Jurjus, G.J., Meltzer, H.Y., Uylings, H.B., Friedman, L., Rajkowska, G., 2004. Cellular changes in the postmortem hippocampus in major depression. Biological psychiatry 56, 640-650. 


\section{Figure legends}

Figure 1. Regions of differences in neural activation during emotional appraisal between A) HC-VAL and MDD-VAL, B) MDD-MET and HC-MET and C) MDD-MET and MDDVAL; FDR whole-brain corrected on cluster level with $\mathrm{q}<0.05$. 